\title{
CORRECTIONS TO THE IAU 1980 NUTATION SERIES FROM LAGEOS OBSERVATIONS
}

\author{
V.K. TARADY \\ Main Astronomical Observatory \\ Ukrainian Academy of Sciences \\ 252127 Kiev \\ USSR
}

The attempt was made to determine the corrections to the coefficients of the IAU 1980 nutation series together with other geodesical and geodynamical parameters by the use of the KievGeodynamics-3 program complex for LAGEOS laser data analysis. This complex is based on the MERIT standards and on numerical integration techniques for satellite orbit computation. An integration is carried out in cartesian coordinates by the Adams method of variable step and order.

At the first stage of the data analysis the 3-minute normal points of ranges to LAGEOS (laser observations from 1986-1987) were used for deriving the orbital elements and station coordinates.

Then at the second stage the Earth rotation parameters and the orbital elements were determined on the basis of the 5-day arcs.

Finally, using the arcs of about 14 days the following parameters were adjusted: Earth rotation parameters; fortnightly nutation term; Love numbers $h, k, l$; geocentric constant of gravitation, etc.

The final r.m.s. residuals of the orbital fits were about $8 \mathrm{~cm}$. We have found the following corrections to the 13.7-day nutation term:

in longitude: $\quad-0.00047 \pm 0.00013$

in obliquity +0"00009 \pm 0.00002 .

\section{Discussion}

HE: (1) What kind of approach is used for the solution of the LAGEos orbit from 1986-1987-a single long arc or multiple short arcs?

(2) Did you include the changes of station coordinates due to the motion of tectonic plates?

TARADY: (1) A multiple short arc pproach was used for the solution of the LAGEOS orbit from 1986 to 1987. In the procedure of ERP determinations we have used 5-day arcs, but for the determination of short-period nutation we have taken 13.7-day arcs.

(2) The changes of station coordinates due to the motion of tectonic plates were taken into account in accordance with the MERIT standards using the AMO2 model. 